\title{
Multimodal Music Composition
}

\author{
Joanne Armitage and Kia Ng \\ Interdisciplinary Centre for Scientific Research in Music (ICSRiM) \\ School of Music and School of Computing \\ University of Leeds, Leeds LS2 9JT, UK \\ info@icsrim.org.uk,www.icsrim.org.uk
}

\begin{abstract}
Multimodality is inherent in the majority of our media experiences, most commonly exemplified in the cinematic arts. A combination of two (or three) dimensional video, dialogue, audio, soundtrack, and occasionally, vibrating chairs or emotive smells, create an engaging environment that submerges audience members into the action. Despite our continuous engulfment in stimulating environments, it can be difficult to isolate how sensory information effects our perception of an experience. By understanding an individual's perceptual and emotional response to multisensory experiences we can harness communication to enhance user experience. This paper discusses multimodal and multimedia technology communications that can enhance music composition by incorporating additional sensory stimulation. First the work's context is given, followed by previous work that has informed this project. The paper presents design concepts, developments and considerations, concluding with the current status and future directions.
\end{abstract}

Multimodal. Touch. Haptic. Composition. Music. Augmentation. Sensory.

\section{INTRODUCTION}

Our senses are physiological tools that allow us to decode, or perceive and then respond to, or feel our surroundings. Consequently, the word 'sense', has two meanings as a verb; firstly as a, 'faculty of perception', and then as a, 'meaning or interpretation' (Harper 2012). Gibson (1966) famously discusses the former when considering the senses as a perceptual system. As defined by Aristotle, in De Anima, human beings have at least five exteroceptive senses (vision, hearing, smell, touch and taste), primarily detected through the corresponding sensory organs (eyes, ears, nose, skin and tongue). In this paper, the perceptual system is considered a tool through which sensation occurs, both physically and emotionally.

Perception occurs through stimulation of sensory neurons that transmit messages to the central nervous system. The intricacies of these messages are specific to each sensory domain. In our detection of the environment, no single sense can convey enough information to truly relay our surroundings. To use the body effectively within the environment the senses work interdependently. Sensory integration, as summarised by Ayres (2005), is "the organisation of sensations for use", or the procedure by which the brain processes multiple sensory modalities to produce meaningful information.
In their most crude form art, music and dance could be considered to focus on the visual, auditory and somatic sensory domains, respectively. However, much like the senses themselves, these art forms are highly integrated and interdependent. For example, a simple orchestral performance has strong visual elements from the movement of the conductor and performers, to the architecture of the performance environment. It is elementary that our perception of sound waves is a somatic response to vibration, enhanced through the tactility of the ear. Further to this, the physical sensations of sound are imparted on the both the body as a whole, and objects in the performance environment.

Metaphorically, music is said to impart a visceral impression upon the body. Such emotional responses are key to the success of composition and musical performance practice. There are many theories encompassing musical aesthetics and psychology that attempt to qualify a listener's literal and metaphorical response to music. However, quantifiable perceptual measurements, particularly in relation to multimodal sensory stimulation in performance, are difficult to obtain. An extension of this is a complex dissection of the individual sensory components of a musical performance and their direct and indirect effect on the listeners' emotion. 
Musical composition focuses on the listener's auditory perception of sonic events. Therefore, the composers primary concern is to define musical parameters such as pitch, timbre, duration, loudness and location. The score provides a means of visually communicating these parameters to the performer. Depending on their interpretation, a particular sound is produced. Sensitive and delicate structures within the ear allow the listener to perceive sound. The human ear is limited in its perception of frequency and sensitivity to loudness. These psychoacoustic boundaries place limitations on the composer in terms of the listeners' perception and cognition of musical events. How can the composer obtain greater control of visceral and metaphorical experiences in music through extra-sensory stimulation?

Overall, the aim of this project is to model the relationship between human perception of sonic, visual and somatic to be utilised in the augmentation of musical expressions through a series of technology-enhanced compositions. In this paper, we propose a means of incorporating additional multimodal channels of communication through which, musical expression and emotional intensity can be conveyed to the listener.

This paper is structured as follows: Section 2 presents a background literature survey incorporating, an overview of the physiology and psychology of touch, haptic systems in accessibility and entertainment, and systems utilising related techniques. Section 3 presents the design and development of the individual system modules, and Section 4 concludes with a summary and current findings.

\section{RELATED BACKGROUND}

Touch is often considered the most complex of the senses, with no localised sensory organ. Philosophically, it has been considered both the most, and least important of the five senses. Invariably, this is due to our reduced awareness of feeling, compared to seeing and listening, and enhanced by the numerous variables that affect it. The human perception of haptic information has been considered historically, as far back as 'The Vedas', an ancient Indian text, and ancient China, where 'five senses' were related to 'five elements' in nature. Numerous haptic perception philosophies have permeated many cultures, with knowledge on the workings of the touch mechanism itself, remaining undisclosed until the last 200 years (Jütte 2008). Nowadays, our increased physiological understanding of touch allows greater exploration of the psychophysical and perceptual applications of tactility, in both media and accessibility focussed applications.
This paper discusses the basic mechanisms involved in touching alongside the basic principles of its perception, and integration with other sensory modalities. A discourse on both historic and current projects is given, encompassing a broad range of haptics in human-computer interactions $(\mathrm{HCl})$ applications, with a particular focus on accessibility, entertainment and music performance.

\subsection{The Sense of Touch}

It is difficult to comprehend how much we physically feel each day; perhaps what is most confusing is considering what we actually do feel. It was not till the mid $19^{\text {th }}$ century that Weber conducted the first systematic physiological experimentation into haptic perception. Concluding, in part, that certain points on the skin were most sensitive to pressure and heat. Further experimentation by predecessors, Blix and Goldscheider, corroborated that there are clusters of receptors in the skin to sense both temperature and pressure. In 1894, Frey confirmed that not only are pressure, warmth and cold each sensed with individual sensory organs, but also an additional, forth sensation, pain. The validity of this and other early experimentation endures to current times (Grunwald \& John 2008).

The basic anatomical receptors, or sensory organs, of touch are as follows: There are four different types of mechanoreceptors located within the skin and subcutaneous tissues. A deeper understanding of these structures can be garnered from Goodwin and Wheat (2008). Receptors, or nerve endings, are located all over the body covering most areas: A notable exception being the brain, which is not sensitive to haptics. As aforementioned, denser receptor clusters are often found in the skin, particularly around hair follicles, but also around joints, muscles, blood vessels and within the ear. Stimulating different combinations of these five primary receptor distributions affects the dimensions of our sensation. In that, a cutaneous sensation, that only affects skin receptors, is different to a more kinaesthetic movement, where receptors in the skin, muscle and joints can be stimulated. This project is interested in the relationship between events stimulating both the haptic and auditory systems. The abovementioned nerves within the ear, specifically the cochlea (the receptive structure of the ear), are essentially sound, or vibrations information pathway to the brain (Gibson 1966). Despite these clear similarities there is limited discourse on the integration of the haptic and auditory. 


\subsection{Haptic Illusions}

Haptic and tactile illusions fall into two predominant categories, those effecting the users perception of physical attributes of an object, and haptic spatial perception. In its extremities, perception itself can be considered an illusion. However, a more accepted definition is given by Lederman and Jones (2011), "an illusion is the marked and often surprising discrepancy between a physical stimulus and its corresponding percept." The neuropsychology of haptic perception is a developing field, particularly in its cognition. A common manifestation of currently unexplained sensory phenomena is the phantom sensations felt by some amputees. Such perceptual illusions are subject to a lesser degree of analysis and understanding than their visual and auditory equivalents. A discussion and analysis of these phenomena can be found in Lederman \& Jones (2011).

\subsection{Haptics and Accessibility}

The application of haptic, or touch based systems increases accessibility for the visually and auditory impaired. Tactile codes such as braille, present a form of written language for the blind or visually impaired. Braille could be considered a form of active touch, or touching, where the environment and surrounding objects are decoded through exploratory feel. Conversely, passive touch, or being touched, presents a tactile communication channel for information to be relayed through stimulating the skin (Gibson 1962).

Touch is an integral sense for the visually impaired. It has been reported that blind people demonstrate increased tactile acuity; understandably, there is much controversy in these conclusions. Goldreich and Kanics (2003) found that tactile perception was considerably increased in blind subjects. They concluded that variables such as age, gender and pressure affect a subject's tactile acuity. Similar conclusions are drawn by earlier research into the tactile perception of Braille readers, by Van Boven et al. (2000). Contradictory research includes that of Grant et al. (2000), who concluded that with practice, sighted subjects could perform three tactile tasks comparably to the visually impaired.

Some haptic technology systems specifically focus upon increasing accessibility for the visually impaired. Koslover et al. (2012) developed a mobile navigation device that outputs visual, audio and vibrotactile cues. They found that navigation cues could be effectively reconstructed through multiple actuators placed on the torso. Hao \& Song (2010) also designed a system to present situation awareness information. Users interpret vibrotactile information from twelve different actuators around their torso.

There is significantly less discourse concerning the tactile perception of the deaf and hearing-impaired in neuropsychological research. Van Dijk (2013) conducted a study to compare the active touch of the deaf and a hearing control group, concluding that those with sign language proficiency outperformed other groups. Famously, the worldrenowned, profoundly deaf, percussionist, Evelyn Glennie, hears music through feeling sound. As her perfect-pitch hearing deteriorated, she trained her body to identify the pitch of notes. Glennie (2003) discusses this stating: "Hearing is basically a specialised form of touch."

Haptic technology systems have been designed, with a focus on enhancing the listening experience for the hearing-impaired. For example, Nanayakkara's (2009) haptic chair explored the distinction between auditory, visual and the somatic sensory modalities. Speakers are integrated into the chair to physically recreate the vibrations of the audio. Vibrotactile feedback, together with synchronised visuals, augments the music listening experience for the hearing-impaired. He focused on congruent reconstructions of sound; the auditory and vibrotactile waveforms are analogous. With this research project, the psychoacoustic properties of sensory interaction will be explored. Counteracting, incongruent attributes of the modalities will be exploited to produce effects that will further understanding of human perception of somatic stimulation.

\subsection{Haptic Systems and Applications}

Haptic technology systems focus on relaying information to users via stimulation of the skin. These technologies are commonly integrated in both consumer and research environments. In consumer electronics, products such as mobile phones and games controllers often incorporate some element of haptic feedback. Mobile phones are the most prevalent haptic devices, assimilating a small actuator that vibrates the device when, for example, a call or text message is received. Games controllers such as the DualShock 3, developed by Sony, include haptics, enhancing user immersion in the gaming environment.

The integration of tactile stimulation into an entertainment environment is not a novel concept. In 1959, director William Castle introduced the 'Percepto' seat alongside his film The Tingler. The seats were built from vibrators salvaged from World War II aircraft and activated at random by the projectionist in certain scenes of the movie (Browne \& Browne 2001). The concept endured through smaller scale productions in amusements parks. In 
the United Kingdom, there are plans to introduce '4D' vibrating chairs in thirty cinemas across the country.

Research in the field of haptics and entertainment predominantly focuses on user experience enhancement. Tactile displays are often the basis of such research due to their size, portability and relatively low cost. Disney research has successfully implemented a 'Surround Haptics' algorithm, that utilises some of the haptic illusions alluded to previously. Application of such techniques allows a lower resolution display $(4 \times 3$ actuators) reducing device cost and intrusiveness. The vibrations produce a smooth tactile motion through 'virtual actuators' that can occur between any two physical actuators on the grid. The haptic display is built into the back of a gaming chair (Israr \& Poupyrev 2010). A similar display concept is considered in this project, with a notably different approach to its construction.

Research has been conducted into the application of haptics in musical performance systems. Hayes and Michalakos (2012) propose a system to aid improvised performance through the use of networked vibrating motors. Berdahl et al. (2009) design a system, which uses a thimble to indicate correct fingering position through haptic feedback. The performers could individually adjust the strength of haptic output. There are numerous other implementations of haptics in musical performance, including new interfaces for musical expression and communication of tempo (Armitage et al. 2012).

\section{DESIGN AND DEVELOPMENT}

This section outlines the design and development of the system with a particular focus on implementation and integration of haptic devices into the performance space. Requirements of the system are overviewed, alongside hardware and software development concepts and implementations.

\subsection{System Overview}

The overall system consists of four distinct modules: Media preparations, performance following, synchronisation and mapping, and reconstruction. In achieving this projects aim a method of incorporating additional vibrotactile and visual data into the score is required. This will allow synchronisation of the musical line, with multimodal events. A module is then required to track audio events with sufficient accuracy to allow alignment with score and triggering of vibrotactile and visual cues. This will involve two methods of performance following including conductor gesture, and real-time audio analysis.

Once the gestural and acoustic data has been captured, the data streams must be synchronised. A set of mapping strategies is required in order to translate the data within the defined parameters of each feedback method. Software and specifically designed hardware for the haptic feedback, is then required to reconstruct the data into both vibratory and visual accompaniments.

\subsection{Design}

This section presents an overall system architecture (see Figure 1) and provides an insight into the key modules.

\subsubsection{Representation}

Augmentation of the musical score in this context requires it to be linked to both vibrotactile and visual events. This requires the information to be in a highly multimedia format. Optical music recognition techniques (OMR) can be applied to the score, converting it to a machine-readable representation with additional multimedia capabilities ( $\mathrm{Ng}$ 2005). At this stage, other events and cues can be added to the score including triggers for the additional modalities. The ISO MPEG Symbolic Music Representation (MPEGSMR) is an exciting development that enables the realisation of the full system, offering better integration of musical scores with multimedia content and applications.

\subsubsection{Score Following}

Central to the performance, the conductor's gestures are used to direct all other elements as a cohesive unit. In our application scenario, a performance can be 'followed' either by tracking and analysing the conductor, and/or 'following' the resultant audio. For our system, the conductor's gestural data is currently captured using the mConduct baton (Armitage et al. 2012), a multisensory interface to capture the hand gestures of conducting. Integration of an inertial measurement unit (IMU) into the base of the baton captures three-dimensional accelerometer, gyroscope and magnetometer data. The IMU measures 3D vectors depending on its orientation. The effect of gravity on the accelerometer is calculated using quaternions, to find global movement (Madgwick 2010). Congruently, the actual sound produced by the ensemble is analysed in real-time using microphones and a fast Fourier transform algorithm. These data streams are then integrated and synchronised facilitating a multimodal approach to score following. 


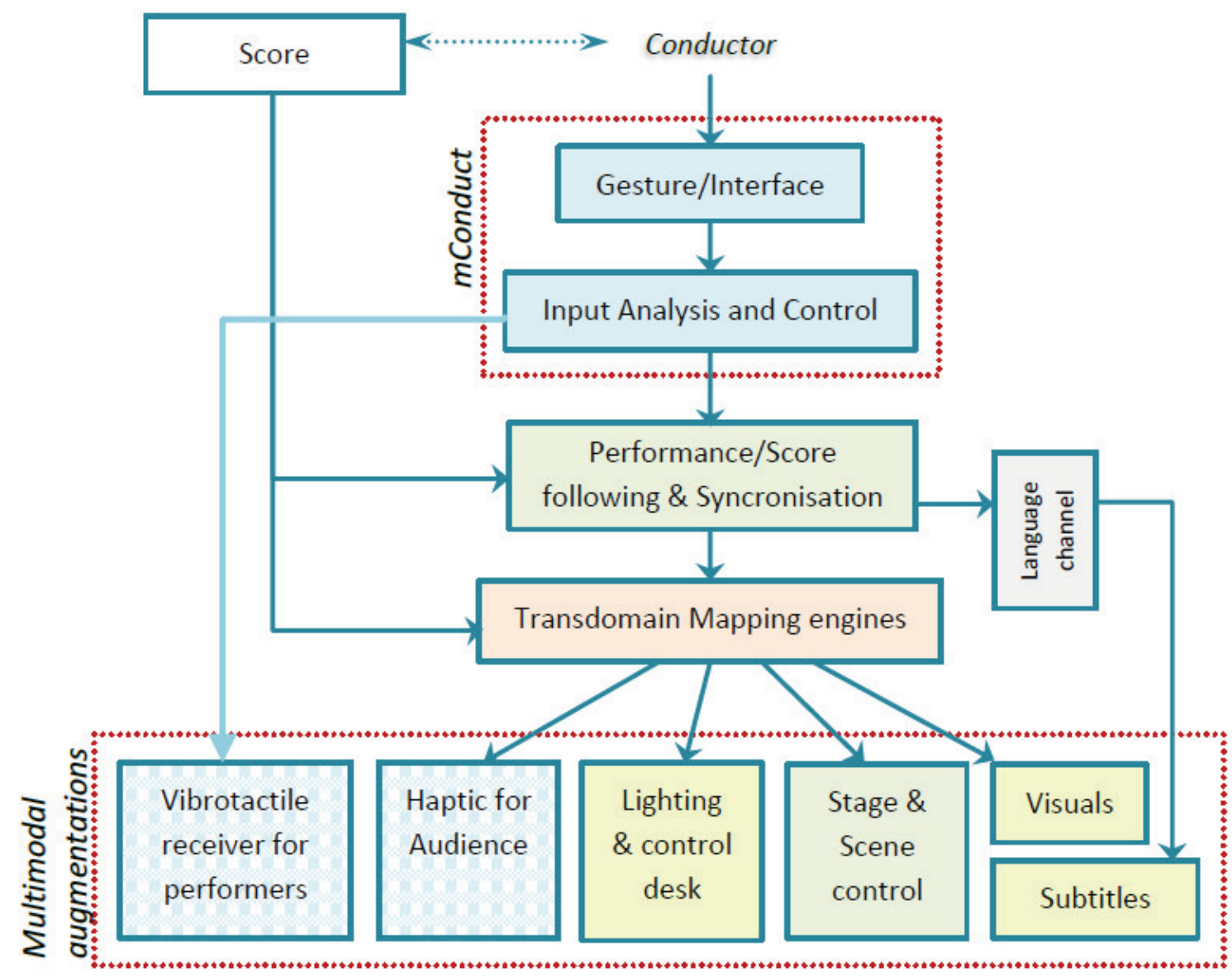

Figure 1: An overall architecture of the system

\subsubsection{Vibrotactile Reconstruction}

This paper considers the implementation of haptic feedback for the listener in a live musical performance environment. A configurable and extensible transdomain mapping strategy is currently being developed for the vibrotactile reconstructions. The system is designed to facilitate haptic reconstructions preprogramed and triggered by the composer, as well as functionality to produce ad lib. reconstructions for pre-existing auditory work. At this stage there are several parameters of vibration that can be controlled by the system including frequency, duration, amplitude, waveform and location. Combinations of these parameters are fine tuned to specific auditory and haptic relationships in order to enhance expressivity of the musical accompaniment.

A software algorithm is currently being designed to control the actuator output. This project is particularly interested in illusions, or psychophysical phenomena, that can enhance user experience through both intensifying emotions and reducing hardware requirement. Vibrotactile or tactile apparent movement, or motion (VAM) is defined by Niwa et al. (2004), "When activating two or more tactors sequentially with a certain timing, the stimulation point is perceived as if it is moving continuously from one position to another, although the physical stimulating points are discrete." Alongside other illusions such as cutaneous saltation, VAM is of particular interest to this project, particularly in its implementation on a tactile display. We are currently trialling an implementation of VAM, with coin-type Eccentric Rotating Mass (ERM) motors.

Hardware is required to physically translate the control signals into haptic feedback. This consists of a two-dimensional array of actuators. We are currently refining the design of the haptic interface with two different approaches. As discussed in Section 2, the actuators can be integrated into the back of a chair, or a 'haptic chair' (see Figure 2). However, a more novel construction is also being considered, with a 'haptic backpack', where the actuators are integrated into a backpack. The later, allows greater portability and flexibility in audience member movement. Furthermore, audience members can passively feel the vibration on their torso, or actively explore the display reconstruction with their hands. Further validation procedures are currently underway for both the system as a whole and the individual components. A particular focus is upon perceptually measuring the user's response to haptic stimuli when combined with audio, and the 
nature of a user's organic and learned response. Establishing psychophysical correlates between musical and haptic motifs and gestures.

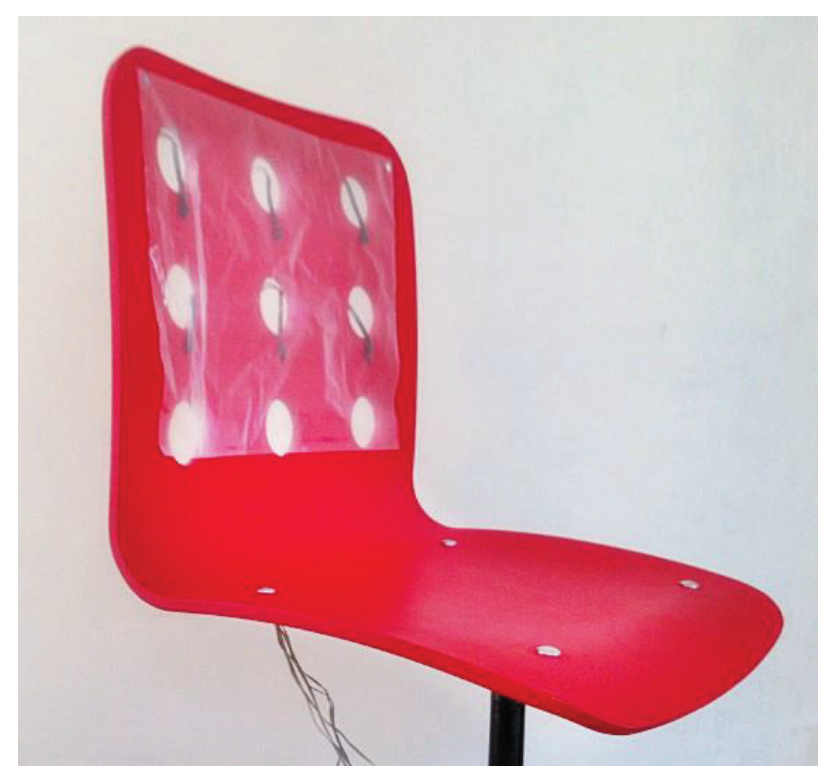

Figure 2: Initial prototype for a portable haptic chair back

\subsubsection{Other Media Reconstructions}

The modularity of the system allows the performance to be translated from an audio experience to other forms of representation including visual. This augments the performance and widens accessibility for greater appreciation and enjoyment of composition and musical performance. In the context of a larger scale work, such as an opera or musical theatre performance the data stream can also be used to control supertitles, change scene, and trigger additional multimedia content including backdrop projections and lighting (see Figure 1).

\section{CONCLUSION}

Sound propagates through space as a series of vibrations; the physical attributes of this motion excite and engage listeners on another level. For example, when standing close to a loudspeaker, you can feel the propagation of waveforms through the speaker cone; this is particularly prominent at low frequencies. Instrumentalists feel parallel, physical sensations as their instrument produces sound. Haptic and vibrotactile stimulation can provide a further sensory modality, or channel of communication through which musical information can be enhanced.

For this project, sensory augmentation is applied to music composition using vibrotactile and visualisation techniques with a view to expanding the spectrum of emotive communications for new musical expression. Integrating these technologies into a musical performance allows the composer greater control of both the compositional outcome and performance environment. With the increase in music listening through digital media, the visceral qualities of sound are less apparent. Somatic reconstructions of sound have the potential to enhance expression in both analogous and digital media.

This paper considers the use of haptics in live music performance to enhance immersion, emotion and expression to audience members. A survey of related literature has been presented, including discourse relating to the sense of touch from both physiological and psychological perspectives, the use of haptics in entertainment and accessibility applications, and the integration of multimodal techniques into music performance. An overall view of the system is given, alongside an analysis of current hardware and software considerations for the vibrotactile audience system. Building upon current research into human sensory perception and psychophysical phenomena and controlled by small vibrating actuators with varying frequencies, amplitudes, waveforms and configurations. This project is only touching the surface of the broad and exciting possibilities within haptic technology. As both knowledge and hardware research expands, so will the potential and impact of such systems. Enhancing awareness through visual and haptic sensations goes beyond the arts, with particular implications in accessibility and wellbeing. Physical reconstructions of sound allow the hearing impaired greater awareness of their surrounding sonic environment. Possible realisations of this include navigation systems, physiological monitoring systems and human computer interfaces. The addition of haptic technology into headphones could allow the user to experience the physicality of sound at lower amplitudes.

\section{REFERENCES}

Armitage, J., Bakanas, P., Halpin, P., Hudspeth, K., Ng, K. (2012) mConduct: A Muti-Sensor Interface for the Capture and Analysis of Conducting Gesture. Electronic Visualisation and the Arts (EVA 2012), London, pp. 229-236, British Computer Society, London.

Armitage, J., Bakanas, P., Balmer, J., Halpin, P., Hudspeth, K. and Ng, K. (2012) mConduct: Transcending Domains and Distributed Performance. International Computer Music Conference (ICMC 2012).

Berdahl, E., Niemeyer, G. and Smith, J. O. (2009) Using haptics to assist performers in making 
gestures to a musical instrument. New Interfaces for Musical Expression (NIME 2009).

Browne, R. B. and Browne, P. (2001) The Guide to United States Popular Culture. Wisconsin Press, Madison, WI.

Gibson, J. J. (1962) Observations on Active Touch. Psychological Review, 69(6), pp. 477-491.

Gibson, J. J. (1966), The Senses Considered as Perceptual Systems, Photolithography Unwin Brothers Ltd., London.

Glennie, E. (1993), Hearing Essay, http://www.evelyn.co.uk/resources/essays/hearing \%20essay.pdf (Apr. 2013).

Goldreich, D. and Kanics, I. M. (2003) Tactile Acuity is Enhanced in Blindness. Journal of Neuroscience 23(8) pp. 3439-3445.

Goodwin, A. W. and Wheat, H. E. (2008), Physiological mechanisms of the receptor system. In Grunwald, M. (eds.). Human Haptic Perception: Basics and Applciations, Birkhäuser, Berlin.

Grant, A. C., Thiagarajah, M. C. and Sathian, K. (2000) Tactile Perception in Blind Braille Readers: A psychophysical study of acuity and hyperacuity using gratings and dot patterns. Percept Psychophys 62(2) pp. 301-312.

Grunwald , M. and John, M. (2008), German pioneers of research into human haptic perception. In Grunwald, M. (eds.). Human Haptic Perception: Basics and Applciations, Birkhäuser, Berlin.

Hao, F. and Song, A. (2010) Design of a vibrotactile display waistbelt. World Congress on Intelligent Control and Automation, (WCICA 2010), pp. 5014-5017.

Harper, D. (2012), Etymology Online: Sense, tp://www.etymonline.com/index. php?term=sense (Mar. 2012)

Hayes, L. and Michalakos, C. (2012) Imposing a networked vibrotactile communication system for improvisational suggestion. Organised Sound, 17(1), pp. 36-44.

Israr, A. and Poupyrev, I. (2010) Exploring Surround Haptics Displays. Human Factors in Computing Systems (CHI 2010), pp. 4171-4176, ACM Press.
Jean Ayres, A. (2005), Sensory Integration and the Child, Western Psychological Services, Los Angeles, CA.

Jütte, R. (2008), Haptic perception: an historical approach. In Grunwald, M. (eds.). Human Haptic Perception: Basics and Applciations, Birkhäuser, Berlin.

Koslover, R. L., Gleeson, B. T. and de Bever, J. T. (2012) Mobile Navigation Using Haptic, Audio, and Visual Direction Cues with a Handheld Test Platform. IEEE Transactions on Haptics, 5(1), pp. 33-38.

Lederman, S. J. and Jones, L. A. (2011) Tactile and Haptic Illusions. IEEE Transactions on Haptics, 4(4), pp. 273-294.

Nanayakkarai, S. C. (2009) Enhancing Musical Experience for the Hearing-Impaired Using Visual and Haptic Displays, University of Singapore.

Lim, K. A., Raphael, C. (2010) InTune: A System to Support an Instrumentalists Visualization of Intonation. Computer Music Journal, 34, pp. 45-55.

$\mathrm{Ng}, \quad$ K., (2011) Interactive Multimedia for Technology-Enhanced Learning with Multimodal Feedback, in Musical Robots and Interactive Multimodal Systems, Springer Tracts in Advanced Robotics, 74, pp. 105-126,

Madgwick, S. (2010) An efficient orientation filter for inertial and inertial/magnetic sensor arrays. Technical Report, Department of Mechanical Engineering, University of Bristol.

Ng, K. C. (2005) Optical music analysis for printed music score and music handwritten manuscript. In George, S. (ed.), Visual Perception of Music Notation.

Niwa, M., Yanagida, Y., Noma, H., Hosaka, K. and Kume, Y. (2004) Vibrotactile apparent movement by DC motors and voice-coil tactors. International Conference on Artificial Reality and Telexistence (ICAT 2004), Seoul, Korea, pp. 126-131.

Van Boven, R. W., Hamilton, R. H., Kauffman, T., Keenan, J. P. and Pascual-Leone, A. (2000) Tactile Spatial Resolution in Blind Braille Readers. Neurology, 54(12) pp. $2230-2236$.

van Dijk, R., Kappers, A. M. L, and Postma, A. (2013) Haptic Spatial Configuration Learning in Deaf and Hearing Individuals. PLoS ONE 8(4): e61336. doi:10.1371/journal.pone.0061336. 